\title{
基于 DEFORM 的模具设计优化
}

\section{Mold Design Optimization Based on DEFORM}

李兵

Bing Li

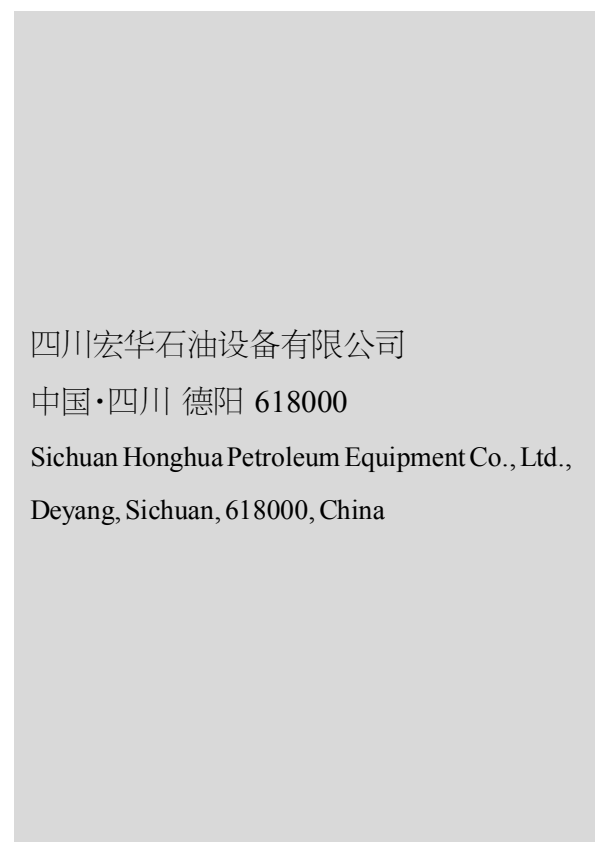

【摘要】在现代材料成型技术中,使用 $\mathrm{CAE}$ 软件进行成型过程模拟, 已成为发展趋势。 CAE 技术通过将工程实际转化为数字模型, 进行计算、预测, 能在虚拟情况下预测成形中 各工装及工艺的正确性, 并给出重要的技术参数。论文通过 DEFORM 软件对吊环产品小 端成型过程的模拟, 确定在成型良好的情况下的小端重量, 预测了在成型过程中的应力最 大值, 以及模具的失效位置。

【Abstract】In modern material molding technology, using CAE software for forming process simulation, has become the developing trend of CAE technology into digital model, through the engineering practice to calculate predicted that can predict forming in the virtual conditions of each tooling and process in the correctness, and important technical parameters of the paper is given by DEFORM software to small end rings products forming process simulation, determine the small end of the under the condition of forming good weight, predicting the maximum stress in the molding process, and the die failure location.

【关键词】锻造模具; 制坏; DEFORM 模拟; 成形

【Keywords】forging mold; preforming; DEFORM simulate; forming

【DOI】10.36012/peti.v2i1.1298

\section{1 引言}

金属塑性成形(塑性加工或压力加工)是一种重要的金属 材料加工方法,在机械制造、冶金等部门中应用广泛。金属塑 性成形过程十分复杂,基于经验、试验的传统方法分析成形过 程十分困难。随着有限元数值模拟即仿真技术在金属塑性成 形中的应用及快速发展，塑性成形过程分析已不再困难。 DEFORM 是在一个集成环境内综合建模、成形、热传导和成 形设备特性,并基于工艺模拟系统的有限元分析软件。可以模 拟热处理和金属成型分析,包括自由锻、模锻等。通过模拟可 以预先判断模具设计及工艺过程的问题, 并提供重要的技术 参数, 然后针对问题提出相应的解决办法, 保证产品质量达到 工艺设计要求。

\section{2 吊环小端模具优化}

目前吊环小端的生产工艺过程为: 成形 $\rightarrow$ 去毛边 $\rightarrow$ 加 热 $\rightarrow$ 压趐, 通过分析提出了吊环小端直接成形的方法, 工艺过
程改为: 成形 $\rightarrow$ 去毛边, 这样既减少加热火次, 同时也减少压 崤工序, 同时压榃高度保证了一致, 提高配对精度, 节约生产 成本 ${ }^{[1]}$ 。以吊环小端模具为例, 说明了通过三维建模可以对目 前在用模具及优化后模具进行建模(如图 1 所示)。

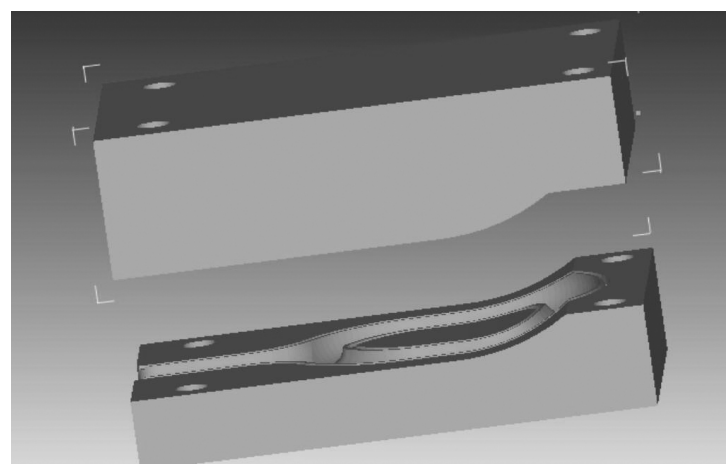

图 1 模具设计

主要改进部位为压趐部位; 由水平分模改为曲面分模; 分 模面选择了中心曲面分模, 保证产品能顺利脱模; 加厚上模, 保证足够的强度及延长模具的使用寿命。材料选择 $5 \mathrm{CrNiMo}$, 
设计制造 Design and Manufacture

通过热处理保证强度, 机械加工后用样板和卡尺对模膛进行 检验。

\section{3 吊环小端改进后的模具成型模拟}

将三维模具转化为 STL 文件, 然后导入 DEFORM 软件, 设置各种模拟参数, 设置成型温度为 $1180^{\circ} \mathrm{C}$, 启动模拟。

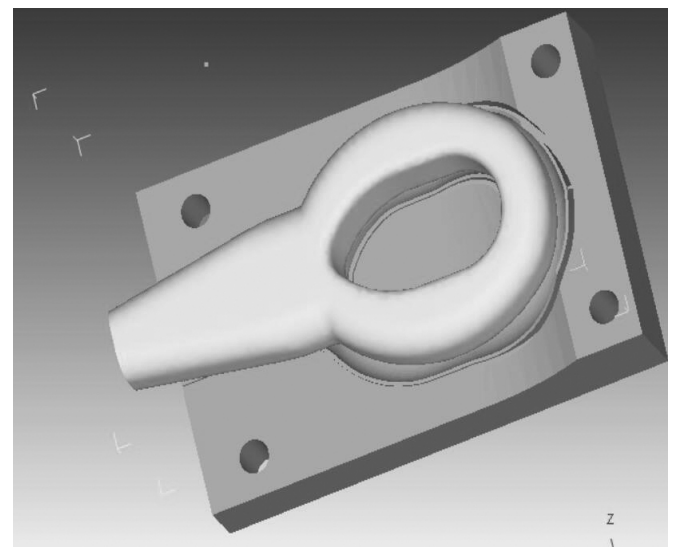

图 2 坏料成型前

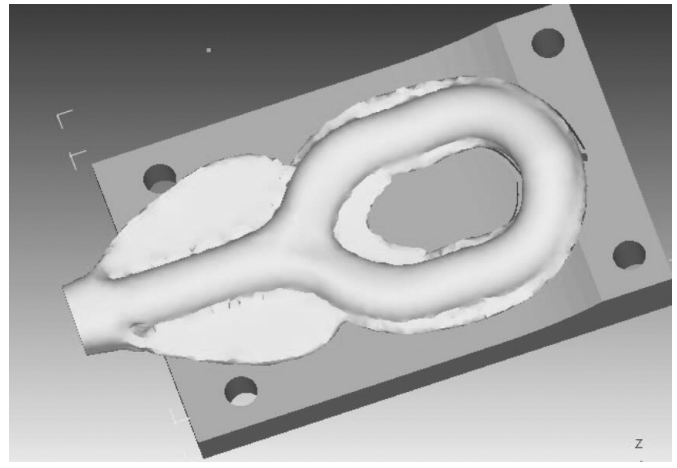

图 3 坏料成型后

坏料成型效果良好,杆部与环部成形效果理想,杆部位置 毛边少且能成形良好，达到吊环成型效果（如图 2、图 3 所 示)。成形良好的环部坏料重量为 $80 \mathrm{~kg}$; 实际工程中, 重量为 $82 \sim 83 \mathrm{~kg}$, 由于工程实际中需要成形两次, 误差 $3 \%$, 预测值与 工程实际符合。

\section{4 模具应力曲线}

使用 DEFORM 后处理器，显示模具应力及坏料变形应 力。最优毛坏成形后,通过软件生成模具应力曲线,生成毛坏 成形时的应力值。图 4 显示了模具应力及毛坏成形时的各处 应力值。模具在 $5 \mathrm{~s}$ 接触毛坏, 应力值逐步上升, 大部分成形时 应力为 $255 \mathrm{MPa}$, 在毛边位置为 $298 \mathrm{MPa}$ 。最终成形时, 毛边开 始形成, 随着变形的继续进行, 毛边逐渐减薄, 金属流人毛边 槽的阻力增大。坏料体积大于型槽容积, 因此, 当型槽完全充 满后, 尚需继续压缩至上下模不断靠近, 多余金属全部排入毛
边槽, 以保证高度尺寸符合要求。因此, 在毛边槽形成最大应 力。在模具实际使用过程中,毛边槽是最先失效的部位。

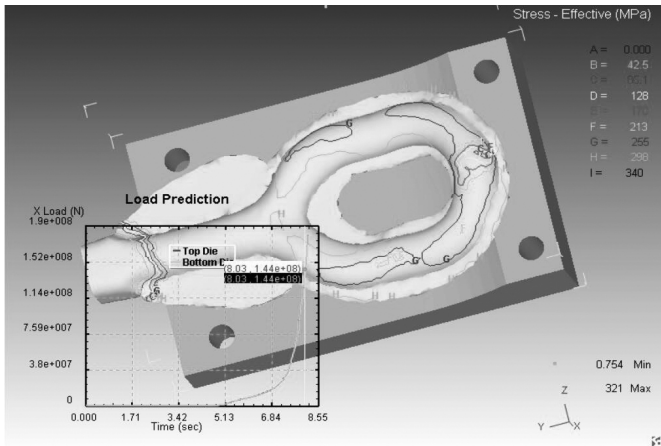

图 4 模具应力及毛坏成形时的各处应力值

因此, 软件预测与工程实际有很好的相符性, 软件能够满 足工艺设计要求, 使用软件模拟能很好地节约成本, 提高产品 质量,减少实物的试制[2]。实物如图 5 所示。

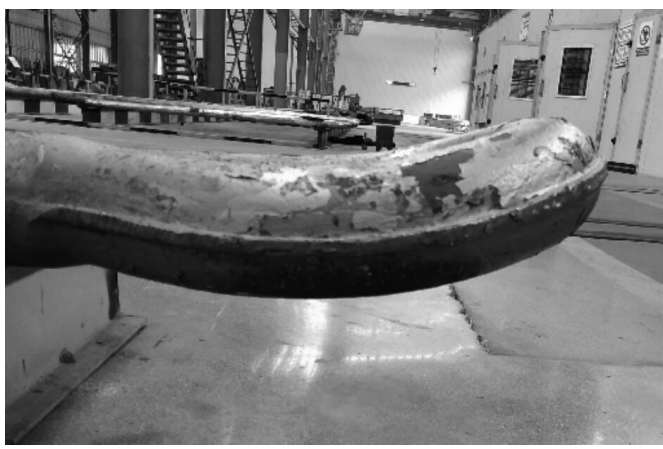

图 5 实物图

\section{5 研究结论}

通过以上模拟研究, 得出如下结论:

(1)通过模拟, 检验了模具设计存在的一个缺陷, 需要对模 具杆部形槽进行倒大圆角 R35 以减轻在模具杆部形槽端部 形成大梯度台阶, 为后续加工提供优质毛坏。

(2)通过四种毛坏成形模拟,优选出最优的成形毛坏(环部 质量 $80 \mathrm{~kg}$ ), 该毛坏满足各处结构尺寸及成型规律。

(3)应力曲线预测了在毛边槽处形成的最大应力, 在最大应 力处是最先失效的部位。这与实际模具使用过程中失效的部位 一致, 因此, 需要在毛边槽处对毛边槽边缘进行加厚优化。

(4)DEFORM 能够很好地与工程实际相符, 完全能满足公 司产品对 CAE 软件的要求。

\section{参考文献}

[1]李忠,付成业,田戊辰,等.基于 DEFORM 的挤压模具设计及优 化[J].工程技术研究,2018(15):3-4.

[2]杨柳. 基于数据驱动的挤压模具工艺参数优化方法研究 [D].广 州:广东工业大学, 2018 . 